determine their functional interplays. Protein-protein interaction assays were performed to detect the interactions of ADAMTS-12 with CTGF

Results: ADAMTS-12-/- mice are more susceptible to collagen-induced arthritis. Accelerated disease onset, significant increase in the arthritis score and arthritis incidence, were observed in ADAMTS-12-/- mice (figure 1a). Histological analysis of whole ankle joints demonstrated a significant increase in synovitis, destruction of bone and cartilage loss in ADAMTS-12-/- mice. ELISA results indicated that ADAMTS-12-/- CIA mice exhibited enhanced release of pro-inflammatory and reduced secretion of anti-inflammatory cytokine. Collectively, these data demonstrate that ADAMTS-12-/- renders mice highly susceptible to CIA.

ADAMTS-12 interacts with and cleaves CTGF, and ADAMTS-12-mediated signalling depends on CTGF during inflammation. It is known that CTGF plays a pro-inflammatory role in the pathogenesis of inflammatory arthritis. We co-transfected CTGF and ADAMTS-12 into 293 T cells and found ADAMTS-12 bound to (figure $1 \mathrm{~b}$ ) and digested CTGF (figure 1c). In vivo studies also demonstrated that CTGF was accumulated in the synovium of ADAMTS-12-/- CIA mice. To further determine whether CTGF is a critical regulator of ADAMTS-12 mediated signalling, we generated CTGF deficient Raw264.7. Overexpression of ADAMTS-12 and CTGF deficiency could decrease the activation of inflammatory signalling markers such as NFkb, p38 and JNK in response to IL-1 $\beta$ at a comparable level. More importantly, overexpression of ADAMTS-12 in CTGF deficient Raw264.7 failed to further inhibit the activation of these signal molecules as compared to CTGF deficient Raw264.7 (figure 1f). Taken together, these results suggest that CTGF is a critical regulator of ADAMTS-12 mediated signalling during inflammation.

Blocking CTGF attenuates inflammatory arthritis in ADAMTS12-deficient CIA mice model. To determine whether the accelerated inflammation in ADAMTS-12-/- mice resulted from the accumulated CTGF, we administered CTGF antibody to ADAMTS-12-/- CIA model after disease onset. The arthritis score in ADAMTS-12 deficient mice was significantly reduced in presence of CTGF antibody (figure 1d). Moreover, histological analysis indicated CTGF abrogated further tissue destruction and inflammation (figure 1e).

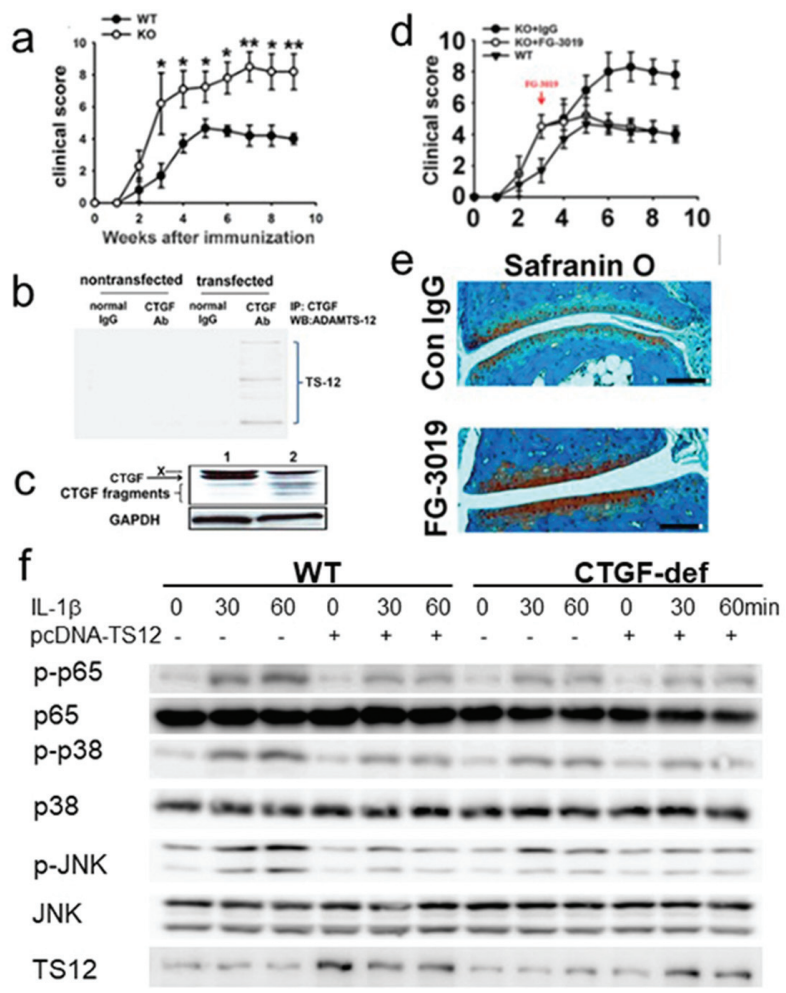

Absatrct OP0224 - Figure 1 ADAMTS-12 protects against inflammatory arthritis through interacting with CTGF. (a) Clinical arthritis scores in WT and KO CIA mice. (b) Co-IP assays detect the associations of ADAMTS-12 with CTGF. (c) Western blotting of CTGF cleavage by ADAMTS-12. (d) Clinical arthritis scores in WT, control IgG-treated KO and CTGF antibody-treated KO mice with CIA. (e) Safranin O staining. (f) WT or CTGF-deficien (CTGF-def) Raw264.7 were transfected with pcDNA-ADAMTS 12 for $48 \mathrm{hrs}$, then cells were treated with or without IL-1 $\beta$ for indicated time. Cell lysate were separated in SDS-page and probed with indicated antibodies.
Conclusions: ADAMTS-12-mediated regulation of inflammatory arthritis is probably through, at least in part, its interplay with CTGF and blockage of CTGF has been shown to be effective in treating inflammatory arthritis.

Disclosure of Interest: None declared

DOI: 10.1136/annrheumdis-2018-eular.4748

\section{OP0225 S100A9 MEDIATES ACUTE NOCICEPTIVE PAIN IN EXPERIMENTAL SYNOVITIS}

A.B. Blom, M.H. Van Den Bosch, E.J. Geven, E.N. Blaney Davidson, P.M. van der Kraan, P.L. van Lent. Experimental Rheumatology, Radboud university medical center, Nijmegen, Netherlands

Background: Synovitis-associated pain is an important aspect of arthritis pathology. Several inflammatory mediators released by the synovium have been implicated in the regulation of pain, including S100A8 and S100A9. These mediators may regulate pain either via direct stimulation of TLR 4 on the nerve endings in the synovium or via stimulation at the site of the dorsal root ganglia (DRG), thereby enabling an increased phagocyte infiltration that may cause sensitisation.

Objectives: To elucidate the role of S100A9 in the pain response after induction of an acute synovitis using streptococcal cell walls (SCW) as a trigger, by comparing S100a9 $9^{-/}$mice and their wild type controls.

Methods: Acute synovitis was induced by a single intraarticular injection of SCW in the knee joint of C57BI/6 (WT) mice and S100 a9-/ mice (also functional knock outs for S100A8). Control mice received a saline injection. Serum S100A8/A9 levels were investigated by ELISA. Joint swelling and cell influx was assessed by ${ }^{99 \mathrm{~m}} \mathrm{Tc}$ accumulation and histology. Pain response were investigated using an Incapacitance Tester (weight bearing), Catwalk (gait analysis) and von Frey's filaments (mechanical allodynia). Gene expression of inflammatory mediators and neuron activation markers in DRG were determined by q-PCR. Monocyte influx and protein expression was monitored by immunohistochemistry (IHC).

Results: A single intraarticular injection of SCW resulted in acute synovitis accompanied by a strongly increased synovial expression of $\mathrm{S} 100 \mathrm{~A} 8$ and S100A9 and increased serum S100A8/A9 levels at day 1, which returned to basal levels at day 7. However, joint swelling and cell influx were similar in WT and S100a9 $9^{-/}$mice at day 1 , excluding a role for $\mathrm{S} 100 \mathrm{~A} 8 / 9$ on pain perception via increased synovitis. WT mice showed a marked and significant decrease in the percentage of weight bearing on the SCW injected hind paw (28\%) compared to saline injection $(47 \%, \mathrm{p}<0.001)$ at day 1 , whereas $5100{ }^{-}{ }^{-}$mice did not. In addition, the stand-phase of the unaffected paws was significantly increased in WT mice 1 day after injection, while in $5100 \mathrm{ag}^{-/}$mice these parameters were not altered. Both mouse strains showed a similar reduction of paw withdrawal threshold, excluding a role for S100A8/9 in allodynia. Analysis of DRG showed no increased phagocyte infiltration after SCW injection and no change in gene expression of the chemokines MCP-1 and KC (for monocytes and neutrophils respectively), and pro-inflammatory cytokines IL-1 $\beta$ and TNF was measured. In addition, F4/80 staining was comparable between WT and S100a9-- mice. However, expression of the neuron activation markers NAV1.7, ATF3 and GAP43 was significantly increased at 1 day after SCW injection in WT mice as compared to saline injected mice ( $p=0.022,0.004$ and 0.030 respectively) while no regulation of these factors was found in $5100 \mathrm{ag}^{-/}$mice, which is in line in with the reduced pain response observed earlier in $5100 \mathrm{ag}^{-/}$mice. The difference in NAV1.7 expression in the DRG was further confirmed at protein level with IHC

Conclusions: These findings show that S100A9 is an important mediator of inflammatory nociceptive pain response in the knee, rather than being involved in peripheral sensitisation. During the acute phase of inflammation S100A8/A9 is likely regulated via direct activation of TLR4 on nerve endings in the synovium and not via monocyte infiltration in the DRG.

Disclosure of Interest: None declared

DOI: 10.1136/annrheumdis-2018-eular.3698

FRIDAY, 15 JUNE 2018

Biologics in RA. More, more and more about safety.

\begin{tabular}{|l|l}
\hline OP0226 & RISK OF HOSPITALISED INFECTION AND INITIATION \\
OF ABATACEPT VERSUS TNF INHIBITORS AMONG \\
PATIENTS WITH RHEUMATOID ARTHRITIS: A \\
PROPENSITY SCORE-MATCHED COHORT STUDY
\end{tabular}

S.K. Chen ${ }^{1}$, K.P. Liao', J. Liu², S.C. Kim ${ }^{1} .{ }^{1}$ Rheumatology, Immunology and Allergy; ${ }^{2}$ Pharmacoepidemiology and Pharmacoeconomics, Brigham and Women's Hospital, Boston, USA

Background: Rheumatoid arthritis $(\mathrm{RA})$ patients receiving biologic therapy are at an increased risk of infection. TNF inhibitors (TNFi) and abatacept are used similarly in RA as the first line biologic. Few studies conducted a head-to-head 